\title{
A Historical Overview of Space Medicine
}

\author{
David R. Williams*, M.Sc., M.D.C.M., F.C.F.P., F.R.C.P.
}

April 12th, 1961 cosmonaut Yuri Gagarin became the first human to fly in space on a one hour and 48 minute mission that orbited the earth. May 5th, 1961 Alan B. Shepard launched from Cape Canaveral on a Mercury Redstone 3 rocket to become the first NASA astronaut to fly in space. These exciting missions started a new era of human space exploration, which over the past 40 years has seen humans walk on the moon to using reusable launch vehicles to construct and live on board space stations on missions lasting from months to greater than one year.

The first decade of human space exploration sought to determine if humans could safely live and work in space. Prior to the Mercury flights, considerable concern existed about the ability of humans to survive in space, as many of the responses to this unique physiological challenge were unknown. Building upon the success of Project Mercury, the Gemini Program demonstrated the capability to rendezvous spacecraft on orbit, to perform space walks, to extend mission duration to be able to support lunar landing and to enhance flight and ground crew operational experience. The decade closed with the historic Apollo 11 mission and the world watched in awe July 20th, 1969, as Neil Armstrong and Buzz Aldrin became the first humans to walk on the Moon.

The second decade of human space exploration evaluated adaptation and performance during long duration missions with NASA astronauts living on the Skylab Space Station. Mission duration ranged from 28 days on Skylab 2 to 84 days on Skylab 4 setting a new record for NASA. Bone loss and muscle wasting were

\footnotetext{
* To whom correspondence should be addressed: NASA Johnson Space Center, 2101 NASA Road 1, Houston, TX 77058, USA.
}

noted in the long duration astronauts despite an orbital exercise program. A lower body negative pressure device was used for the first time to determine the cardiovascular response to a simulated orthostatic stress on orbit. Customary indices of reduced cardiovascular deficiency were obtained. This was found to stabilize after four to six weeks in space with no apparent impairment of crew health or performance. The physiological changes associated with adaptation to microgravity suggested the need for further research to develop countermeasures to mitigate the potentially deleterious physiological changes observed on orbit or after landing.

Following Skylab, Apollo-Soyuz represented a unique technological achievement with US and Russian spacecraft docking to demonstrate international collaboration in space exploration. This collaborative flight was an important precursor to joint operations between NASA and the Russian space program.

The third decade focused on using the first operational reusable launch vehicle, the Space Shuttle, on missions to further our understanding of human capabilities in space and to use the absence of gravity as an important scientific variable to help us understand the role that gravity plays in biological and physical processes on earth. Construction of the Enterprise, a test-bed prototype space shuttle started in 1974 and it was test flown in a series of drop tests from 1977 to 1979 . The first manned test flight of the space shuttle launched April 12th 1981 on a 2-day mission, with a commander and pilot following a rigorous test flight program. Since then NASA has flown more than 100 space shuttle missions to launch satellites, to conduct scientific research in biomedical and microgravity science, to dock with the Russian MIR space station and to build the International Space Station (ISS). 
November 20th, 1998 marked a new era for human space flight as the first element of the ISS, the Zarya Control Module (Functional Cargo Block - FGB) was launched on a Russian Proton rocket. This flight was followed shortly after by delivery of the NASA provided Unity node on a shuttle flight later that year. To prepare the station for habitation, over the next year and a half five flights to the ISS were subsequently launched by NASA and the Russian Space Agency. Commander Bill Shepherd, Soyuz Commander Yuri Gidzenko and Flight Engineer Sergei Krikalev became the first crew to live on board the ISS in November 2000.

Permanent international habitation in low earth orbit required an unprecedented level of cooperation among all of the international partners. While both NASA and the Russian Space Agency had experience with long duration space flight, the ISS brought many new challenges in building and operating what is felt to be one of the most complex pieces of engineering developed cooperatively between five major international partners. Over the next decade the program will make the transition from station construction to utilization providing access to a unique research platform to scientists worldwide. It is anticipated that research conducted on the ISS will help clinicians understand how to optimize the health and performance of astronauts working for prolonged periods in microgravity as well as further scientific knowledge in many areas of fundamental life and microgravity science.

\section{CLINICAL CARE CAPABILITIES}

Extending the time distance constant of human space exploration, keeping humans longer in space and being able to send them to low earth orbit and beyond, has provided a significant impetus to further develop the clinical capability of physicians practicing space medicine. Historically, one of the primary methods of ensuring crew health on orbit has been to maintain a rigorous medical selection and retention process. The clinical capability of providing on orbit medical care evolved from fairly rudimentary support in the early days of the Mercury and Gemini programs to more robust support for the Apollo and Skylab missions. Medical care on board the Shuttle evolved into a series of six pallets that constitute the ShuttleOrbiter Medical System (SOMS) kits. This provided a level of support analogous to a typical primary care setting with medical care rendered by crew medical officers (CMOs) supported by terrestrial flight surgeons in mission control.

Medical care on board the ISS will utilize a crew health care system $(\mathrm{CHeCs})$, made up of three different elements to maintain crew health and ensure safe ISS operations. The environmental health system (EHS) provides in-flight environmental health monitoring of radiation, air and water quality as well as microbial surface contamination. The countermeasure system (CMS) represents a number of different interventions that are used to prevent some of the physiologic changes observed in astronauts participating in long duration space flight. The health maintenance system (HMS) provides on orbit capability to prevent, diagnose and treat ISS crew who are ill or injured thereby minimizing the need for a medical evacuation emergency crew return.

During the early phases of ISS construction on orbit medical care will be provided in a manner very similar to the Shuttle program with additional $\mathrm{CHeCs}$ hardware added incrementally with subsequent missions. Space medical care will be provided by initial intervention from crew medical officers (CMOs) supported by flight surgeons in mission control in a manner similar to the shuttle program. Standardized training and the development of clinical practice guidelines will help ensure optimum health care delivery by crew with differing backgrounds supported on console by international flight surgeons representing many different styles of clinical practice. An evidence based review of diagnostic and therapeutic procedures for on orbit medical care has been an important adjunct in determining current clinical capability and directing future research and development programs.

\section{THE PHYSIOLOGICAL EFFECTS OF MICROGRAVITY}

Understanding the physiological adaptation of humans to partial and microgravity environments has been a critical element in the development of countermeasures to prevent some of the biomedical effects of living in space. There are many physiological changes associated with space flight, some of which occur prior to launch, that have been well described in the literature.

During the seven-day pre-launch quarantine, astronauts often undergo circadian shifting to align their work/sleep cycle with launch and orbital operations. Bright lights and dark glasses are used to facilitate shifting and the potential benefit of melatonin is being studied. The crew typically enters the space shuttle approximately 2 hours prior to launch wearing the advanced crew escape (ACES) suit and are strapped in to complete final pre-launch checklists. Throughout this period the crew are lying horizontal with their hips and knees flexed. With the feet and legs elevated, the normal gravitational 
gradient affecting cardiac return is reversed resulting in a volume redistribution similar to that observed on orbit.

The physiologic adaptation to microgravity begins following main engine shutdown. The predominant immediate changes are cardiovascular and neurovestibular, which typically resolve within 24 to 48 hours. Acclimatization to space takes place over days to weeks with most physiologic systems reaching a new steady state within 4 to 6 weeks on long duration flights. From personal experience on a 16-day space flight, three periods of adaptation were evident. The first was associated with resolution of the symptoms associated with volume redistribution: mild headache, facial fullness and congestion. The next was approximately five days into the mission when it became evident that living and working in microgravity seemed easier. The third occurred around ten days to two weeks into the flight and was associated with feeling totally familiar with the environment having completed the transition from living and working in a predominantly two dimensional framework to fully utilizing the benefits of a three dimensional work space.

Lower extremity muscle wasting and cardiovascular deconditioning is evident with both short and long duration missions. Alterations in bone density are typically associated with long duration space flight with losses of 1 to $1.5 \%$ per month observed. Long duration missions are also associated with decreases in red blood cell mass and increased excretion of urinary calcium. Alterations in the relative populations of white blood cells including neutrophilia, lymphocytopenia, eosinopenia as well as alterations in cell mediated immunity have been observed as well.

Re-entry and landing are predominantly associated with neurovestibular symptoms, varying degrees of orthostatic intolerance, muscle weakness and atrophy as well as cardiovascular deconditioning. Exercise countermeasures on orbit are used to optimize readaptation to life in a gravitational environment yet rehabilitation post-flight is important for both short and long duration astronauts.

It is evident that there are many profound physiologic changes associated with living in microgravity and returning to earth. While research to understand the physiological adaptation of humans to space is critical to developing new countermeasures, it is unlikely that all countermeasures will be completely effective. It is also relevant to consider where research priorities should be placed in developing new countermeasures. Some of the physiologic changes associated with adaptation to space may be acceptable and others may only be detrimental when landing in a partial or earth gravitational environment.

\section{SPACE MEDICINE: CHALLENGES AND FUTURE DIRECTION}

The expansion of human space flight to low earth orbit and beyond over the past forty years has provided a challenge to clinicians responsible for the health and safety of astronauts. Space medicine is currently entering an evolutionary phase of incorporating the understanding of the physiological changes associated with human space flight into the prevention, diagnosis and therapy of illness and injury in space. Applying terrestrial diagnostic and therapeutic approaches to illness in space may be problematic when not only the underlying physiology differs in microgravity; it is likely that the pathophysiology of certain diseases may differ as well.

Surgical capability exists on the ISS but is limited to minor procedures including wound repair. Verification of these procedures has been done on the $\mathrm{KC}-135$, an aircraft used to fly parabolas resulting in approximately 20 seconds of microgravity, resulting in a number of publications on microgravity surgical procedures. On orbit surgical experience, acquired through complex animal dissections on two Spacelab flights, suggests that while the $\mathrm{KC}-135$ is a valuable test bed it is a considerably different environment than the sustained microgravity of space flight. After performing a wide variety of animal surgical procedures on the Neurolab mission, I would have no hesitation in performing minor surgical procedures in space. Experience from the Spacelab missions will be critical in developing the appropriate techniques for surgery in space.

The biomechanics of trauma is quite different in microgravity where objects have mass but no weight, where astronauts may be working inside a spacecraft or performing a space walk where they are protected inside a spacesuit. Working in partial gravitational environments can be associated with injury. As in terrestrial trauma, diagnosis and therapy is dependent upon understanding the biomechanics of injury, an important area for future research (8).

The chain of clinical care for astronauts on missions in low earth orbit extends from immediate care on orbit supported with telemedicine to terrestrial flight surgeons, to stabilization and transfer on an assured crew return vehicle with secondary care at the landing site pending transfer to a definitive medical care facility. This model is acceptable when rapid return to earth is feasible. Extending the distance of human space exploration suggests the need to develop autonomous medical capability on board the spacecraft or planetary outposts. With current technology, the delay in signal transmission will prevent real-time telemedicine limiting terrestrial physicians to providing primarily 
consultative support to their space faring colleagues. The requirement for enhanced autonomous definitive care for such missions will stimulate the development of many new technologies including smart diagnostic systems, minimally invasive keyhole surgical procedures, robotic assist devices for surgical procedures and virtual reality simulators to assist space medicine practitioners. Novel micro- and nanotechnologies will be a key element of the clinical armamentarium of space physicians and nanomedicine a reality of clinical practice.

The operational priority for the next decade will be to develop and utilize the research capabilities of the ISS to enable safe human space travel beyond low earth orbit. The next phase of exploration may involve humans returning to the moon for prolonged periods at lunar habitats, or could involve sending humans to Mars to search for evidence suggesting the existence of life elsewhere in our solar system. If the development of space technology follows the rapid development of aviation over the past century, as we enter this millennium the humans may very well become a space faring society.

\section{REFERENCES}

1. Link MM. Space Medicine in Project Mercury. Washington, DC: Scientific and Technical Information Division, NASA; 1965

2. Mueller GE, ed. Gemini Summary Conference. Manned Spacecraft Center, Houston, Texas: Scientific and Technology Information Division, NASA; 1967.

3. Johnston RS, Dietlein LF, eds. Biomedical Results from Skylab. Washington, DC: Scientific and Technical Information Office, NASA; 1977.

4. Nicogossian AE, Huntoon CL, Pool SL, eds. Space Physiology and Medicine. Philadelphia: Lea \& Febiger; 1994.

5. Sawin CF, Taylor GR, Smith WL, eds. Extended Duration Orbiter Medical Project. Washington, DC: NASA Publications; 1999.

6. Campbell M, Billica R, Johnson S. Animal surgery in microgravity. Aviation, Space and Environmental Medicine 1993; 64: 58-62

7. Campbell M, Billica R, Jennings R, Johnson S. Laparascopic Surgery in Weightlessness. Surgical Endoscopy 1996; 10: 111-117.

8. Kirkpatrick AW, Campbell MR, Novinkov O, et al. Blunt trauma and operative care in microgravity. Journal of the American College of Surgeons 1997; 184: 441-453.

9. Freitas RA. Nanomedicine, Volume 1: Basic Capabilities. Austin, Texas: Landes Bioscience; 1999.

Dr. David R. Williams, MSc, MDCM, FCFP, FRCP, pursued research in neurophysiology before completing medical school at McGill University. He went on to a residency in Family Practice at the University of Ottawa and then to a residency in Emergency Medicine at the University of Toronto. Dr. Williams was selected by the Canadian Space Agency in June 1992. Between April 17 and May 3, 1998, he served as a mission specialist aboard STS-90 Neurolab. During the 16-day Spacelab flight the seven person crew served as both experiment subjects and operators for experiments focusing on the effects of microgravity on the nervous system. Dr. Williams was appointed Director of the Space and Life Sciences Directorate in July 1998. In this capacity he has overall responsibility for research in both physical and biomedical space sciences at Johnson Space Center. Crew medical safety is one of his principal concerns, as are flight medical operations and JSC occupational and environmental health. 